\title{
Another look at diagnostic pathways in children with urinary tract infection
}

\author{
ROBERT H WHITAKER, THOMAS SHERWOOD
}

This is a discussion paper on better ways of tackling a common problem: looking for the cause of a child's first recognised urinary infection. We describe practical choices and programmes adopted jointly in our departments over the past two years. We would be the first to accept that they may not suit all places.

The following factors made us take another look at our diagnostic pathways: $(a)$ in the past few years new light has been shed on our understanding of reflux and reflux nephropathy; (b) it has become increasingly apparent thai an intravenous urogram is no diagnostic panacea in very small children; (c) there are no longer (if indeed there ever were) bottomless funds to pay for expensive investigations for all children with a single urinary tract infection; and $(d)$ the concept that only serious conditions threatening life or the kidney need to be diagnosed on a screening programme.

The first three factors are self explanatory. The fourth might not be universally acceptable. We believe, however, that for the sake of avoiding overinvestigation and containing costs, we can afford to miss the diagnosis of certain relatively minor conditions during the first investigation of a child with infection. This is on the understanding that if the child has a further infection, more conventional investigations will be performed that will pick up even the least worrying conditions.

\section{What are we looking for?}

If 100 children with definite, proved urinary tract infections are investigated by all the means that are available, in our experience about 40 will have some urinary anomaly: roughly 20 will show vesicoureteric reflux, and 20 will have other conditions ranging from relatively unimportant conditions such as misplaced kidneys-for example, pelvic or crossed ectopic-and various types of uncomplicated duplications to the more important conditions such as the complete duplications associated with reflux or obstruction, hydronephrosis, megaureters, bladder diverticula, and stones. At the most severe end of this range are the children with neuropathic bladders and with severe obstruction from posterior urethral valves.

The 20 children with reflux may have a considerable range of disorder. The more severe forms are often found in the neonatal period with massive reflux into poorly contractile ureters. The

Departments of Urology and Radiology, Addenbrooke's Hospital, Cambridge CB2 2QQ

ROBER'T H WHITAKER, MA, FRCS, consultant urologist

THOMAS SHERWOOD, FRCP, FRCR, professor of radiology

Correspondence to: Professor Thomas Sherwood. reflux may be associated with bladder diverticula or may be sufficiently severe to suggest a secondary pelviureteric obstruction. Similarly, reflux may be associated with a neuropathic bladder. The rest of this group consists of primary reflux, commonly graded I to III in its severity ${ }^{1}$ : grade I (slight)incomplete filling of the upper urinary tract without dilatation; grade II (moderate)-complete filling of the upper urinary tract with slight dilatation but no ballooning of calices; and grade III (gross)-complete filling of the upper urinary tract with marked dilatation and obvious ballooning of calices.

\section{Some relevant points in respect to reflux}

Ten or more years ago the conservative treatment of children with reflux was to administer long term antibiotics until the reflux stopped. The fear was that if antibiotics were stopped further infection might lead to progressive or new scarring of the kidneys. This is now known to be so rare, particularly in the older child, that the patterns of conservative management have changed considerably. We now believe that if scarring is going to occur it will happen with the first urinary tract infection, provided that the papillary morphology is such as to allow intrarenal reflux. The damage seems to be initiated rapidly, so that unless treatment with antibiotics is begun within hours of the urinary tract infection starting, the scarring is predestined in any particular kidney. Conversely, scarring is rarely seen as a new phenomenon, particularly in children over the age of 4 years.

The other important factor that has emerged recently from the Birmingham Reflux Study Group and studies at the Hospital for Sick Children in Great Ormond Street is that conservative treatment is just as good as successful reimplantation in terms of breakthrough urinary tract infection, renal function, and new or progressive scar formation. ${ }^{23}$ Reflux preventive surgery is a vexed issue, and it is usually difficult to get two paediatric urologists to agree as to their indication for such surgery. The devil's advocate would suggest that treatment in general has little if any effect on the clinical course of reflux nephropathy unless or until we identify those kidneys in which there is intrarenal reflux before any infection occurs within the kidney. This goal is not attainable even with the invasive techniques presently available.

\section{Investigative techniques}

Before putting forward diagnostic pathways we must consider what investigative methods are available to us and what they can achieve.

Plain abdominal $x$ ray examination-Such an examination can of course identify radio-opaque stones, but it may also suggest an enlarged or poorly emptying bladder, displacement 
of the bowel shadow from the hypochondrium by a large fluid filled kidney, etc.

Ultrasound with or without real time facility can identify the size and shape of the kidneys and bladder. It can also identify a dilated pelvicaliceal system and ureter. It should be performed with the bladder full, and if an anomaly is found it should be repeated with the bladder empty. Ultrasound can therefore point to obstructive lesions and to upper tract dilatation associated with the severer forms of reflux.

Labelled dimercaptosuccinate scintigram-This radionuclide technique provides a functional cortical map of the kidney, quantifying renal tubular cell mass. It is particularly useful for identifying scars of reflux nephropathy and for estimating differential function, provided that there is no obstruction.

Labelled diethylenetriamine penta-acetic acid scintigram-This substance is filtered by the glomerulus and gives a dynamic study similar to an intravenous urogram. It identifies dilatation

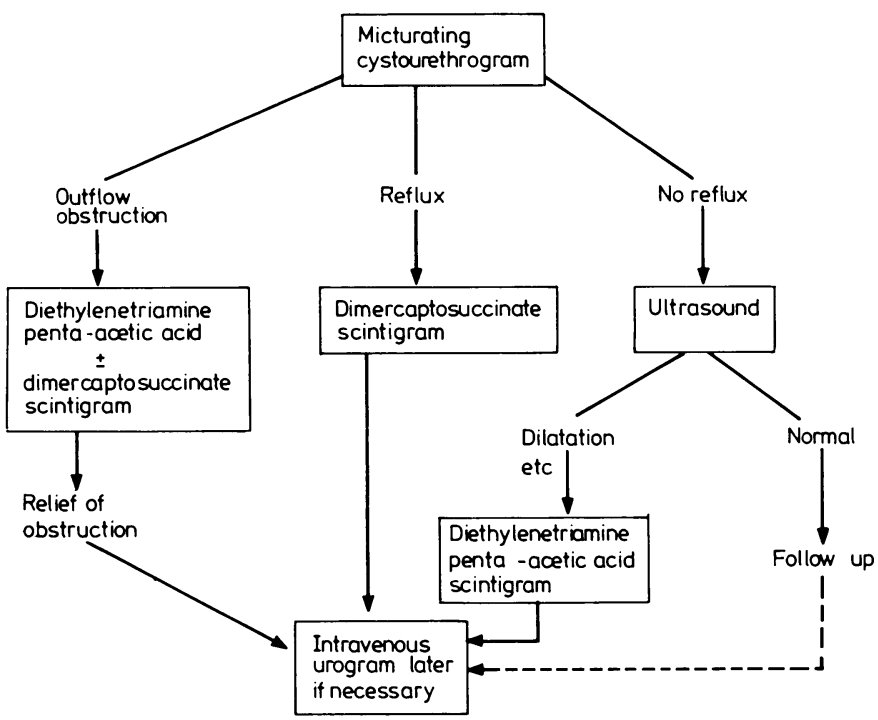

FIG 1-Investigative pathway for children up to 6 months old presenting with first recognised infection.

and obstruction, and in the latter stages of the study it can give information concerning reflux. Differential renal function can also be estimated.

Intravenous urogram-This study is so well established that its benefits do not need to be described, but clearly it gives all the information needed about the morphology of the urinary tract, provided that the kidney is working well enough. Intravenous urograms in young infants are often disappointing and sometimes dangerous.

Micturating cystourethrogram-This study is essential for documenting vesicoureteric reflux and showing abnormalities of the outflow tract. It is also the best means of showing a bladder diverticulum.

Two questions need to be answered. Firstly, how much reflux will be missed if an intravenous urogram only is performed? Previous studies show that minor degrees of reflux will fail to be shown on an intravenous urogram alone, but that no case of grade III reflux will be missed. ${ }^{4}$ We are prepared to accept missing lesser degrees of reflux on initial screening except in very young infants. The second question to ask is how many anomalies will be missed if a plain $x$ ray examination and ultrasound are performed as a single investigative method in an initial screening of children with infection. A separate paper in this issue ( $p$ 829) shows that ultrasound and plain $x$ ray examination give a false negative rate of $10 \%$ so that by this method renal scars can be missed, as can lesser degrees of dilatation and reflux.

\section{Suggested diagnostic pathways}

Armed with the above information and discussions, we have planned the following three diagnostic pathways which vary with the age of the child presenting with a first urinary infection.

Under the age of 6 months (fig 1)-In this group our primary aim is to diagnose degrees of obstruction that threaten the integrity of the kidney and to detect the presence of intrarenal reflux, perhaps before scarring has occurred. Thus we first

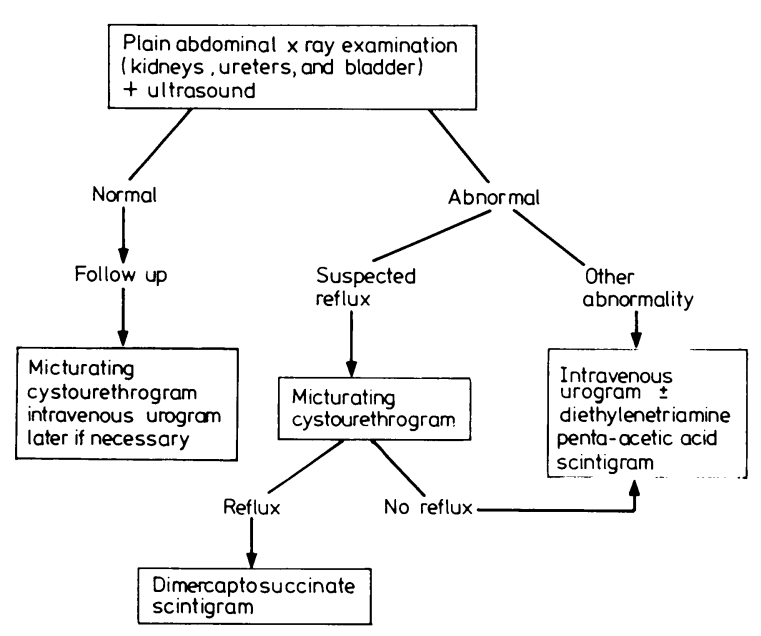

FIG 2-Investigative pathway for children aged from 6 months to 4 years of age presenting with first recognised infection.

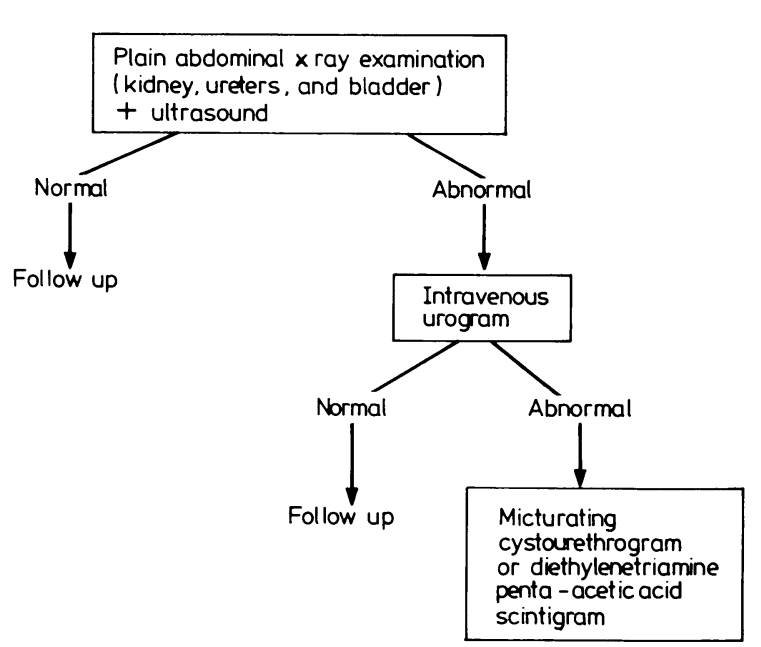

FIG 3-Investigative pathway for children over the age of 5 years presenting with first recognised infection.

perform a micturating cystogram. If this shows outflow obstruction the condition is treated accordingly. If it shows vesicoureteric reflux then the presence or absence of a reflux nephropathy is assessed with a dimercaptosuccinate scintigram, and in due course an intravenous urogram will be performed as a baseline for further investigation. If no reflux is present an ultrasound scan is performed to look for upper urinary tract dilatation or other abnormality; any doubt leads to a study using diethylenetriamine penta-acetic acid to check on urinary drainage and individual renal function. If appearances are normal the child is simply followed up. If abnormal the condition is dealt with accordingly; follow up studies may include a dimercaptosuccinate scintigram, further ultrasound, and possibly an intravenous urogram.

From 6 months to 4 years (fig 2)-All children presenting in this age group undergo a plain $x$ ray examination, an ultrasound 
of the urinary tract, and often a micturating cystogram, though not invariably during the first infection. We appreciate that if the necessary expertise is not available to obtain a high standard of ultrasound then an intravenous urogram is still necessary in this group. If initial studies show no abnormality then the child is simply followed up. If they show abnormality radionuclide tests and other examinations are indicated as for the previous group.

Over 5 years (fig 3) - In this group we believe that progressive damage from reflux nephropathy is most unlikely, so we do not feel the need to diagnose reflux during the first screening procedures. At present in this group we perform an ultrasound and plain $x$ ray examination of the abdomen only. If appearances are entirely normal we follow up the child, and perhaps go on to further investigations at the time of the next infection. If the initial tests show an abnormality the child would undergo either an intravenous urogram or micturating cystourethrogram or both as indicated.

Urinary infection is common in children. Correctable struc- tural causes are few but important. We think that we should make use of new imaging techniques if they are kind and effective, but only to look for what is correctable and important.

\section{References}

${ }^{1}$ Bailey RR. An overview of reflux nephropathy. In: Hodson J, KincaidSmith P, eds. Reflux nephropathy. New York: Masson Publishing USA Inc, 1979:3-13.

2 Birmingham Reflux Study Group. Prospective trial of operative versus non-operative treatment of severe vesicoureteric reflux: two years' observation in 96 children. $\mathrm{Br} \mathrm{Med} \mathcal{F} 1983 ; 287: 171-4$.

${ }^{3}$ Kincaid-Smith P. Reflux nephropathy. Br Med F 1983;286:2002-3.

4 Lanning P, Seppänen U, Huttunen N-P, Uhari M. Prediction of vesicoureteral reflux in children from intravenous urography films. Clin Radiol 1979;30:67-70.

5 Cavanagh PM, Sherwood T. Too many cystograms in the investigation of urinary tract infection in children? Brf Urol 1983;55:217-9.

(Accepted 17 November 1983)

\title{
Mathematics in Medicine
}

\section{Statistical ritual in clinical journals: is there a cure?-I}

\author{
DONALD MAINLAND
}

\begin{abstract}
A disturbing verdict
"The presentation of variability in medical journals is a shambles." This verdict from a Medical Research Council statistician $^{1}$ appeared 55 years after the first edition of R A Fisher's Statistical Methods for Research Workers, the main source of the statistical arithmetic now widespread in medical journals: standard deviations, standard errors of the mean, $r$ 's, significance tests (especially $\chi^{2}$ and $t$ ) with the resulting P's and $p$ 's, ${ }^{*}$ and, more recently, confidence intervals. The verdict seems astonishing because the main reason for using statistical methods is variability; and it is especially disturbing, even conscience pricking, to one who soon after Statistical Methods appeared started applying it to his own research, ${ }^{2}$ mostly using "significance" tests which, to avoid serious ambiguity, should have been called "chance frequency" tests. During 30 years or so after this I tried to propagate the elementary ideas of Fisher's Design of Experiments, which in 1935 presented randomisation as the logical basis for significance tests, to replace the mythical doctrine that a study group and its control should be "alike in all respects" except the factor or factors under investigation.
\end{abstract}

* The author is following Fisher's symbolism where $P$ is used to denote the probability (random frequency) of an observation plus the probability of rarer observations in the same or opposite tail, and $p$ represents the probability of a single class of observations.

Kent Hills, Kent, Connecticut 06757, USA

DONALD MAINLAND, MB, DSC, retired professor of anatomy and medical statistics

Correspondence to: Kent Hills, Apt 3B1, Kent, CT 06757, USA.

\section{Thinking versus arithmetic}

One of the earliest clinical applications of Fisher's Design of Experiments was the trial of streptomycin in pulmonary tuberculosis, which emphasised not arithmetical but statistical thinking - concern with variability and with risks of bias throughout the planning and performance of a study. ${ }^{3}$ After the 'forties controlled trials (of diverse quality) spread widely, but statistical arithmetic spread more abundantly and less rationally; and surveys of medical journals have shown that "in about half of all published articles there are statistical errors," including misuse of arithmetical techniques. ${ }^{4-9}$ Fisher himself came to deplore how often his own methods were applied thoughtlessly as cookbook solutions. ${ }^{10}$ Among reasons suggested for this state of affairs are: emphasis on techniques in elementary textbooks ${ }^{11}$; lack of contact with medical problems during statisticians' training ${ }^{8}$; too much "mathematistry" (development of theory for theory's sake) during the education of consultant statisticians for all aspects of science. ${ }^{12} 13$

In attempts to improve the position the $B M \mathcal{F}$ has issued four series of articles, later published in book form, ${ }^{14-16}$ and a set of guidelines which should help in planning and reporting research $^{17}$; but I wonder whether any of these publications will have much effect on the arithmetical ritual.

\section{A protest}

Referring to the $B M$ ''s instructional articles a letter from a department of pathology asked why one should "try to fit recalcitrant numbers into some analysis which will confirm what one knows in one's bones to be true." 18 The letter quoted two papers in which non-significant differences were apparently counted as "real," and asked: "If we are to be allowed to ignore 